\title{
A class of solitons in Maxwell-scalar and Einstein-Maxwell-scalar models
}

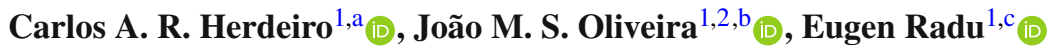 \\ ${ }^{1}$ Departamento de Matemática da Universidade de Aveiro, CIDMA, Campus de Santiago, 3810-183 Aveiro, Portugal \\ ${ }^{2}$ Centro de Matemática, Universidade do Minho, 4710-057 Braga, Portugal
}

Received: 29 October 2019 / Accepted: 21 December 2019 / Published online: 11 January 2020

(C) The Author(s) 2020

\begin{abstract}
Recently, no-go theorems for the existence of solitonic solutions in Einstein-Maxwell-scalar (EMS) models have been established (Herdeiro and Oliveira in Class Quantum Gravity 36(10):105015, 2019). Here we discuss how these theorems can be circumvented by a specific class of non-minimal coupling functions between a real, canonical scalar field and the electromagnetic field. When the nonminimal coupling function diverges in a specific way near the location of a point charge, it regularises all physical quantities yielding an everywhere regular, localised lump of energy. Such solutions are possible even in flat spacetime Maxwellscalar models, wherein the model is fully integrable in the spherical sector, and exact solutions can be obtained, yielding an explicit mechanism to de-singularise the Coulomb field. Considering their gravitational backreaction, the corresponding (numerical) EMS solitons provide a simple example of self-gravitating, localised energy lumps.
\end{abstract}

\section{Introduction}

Einstein-Maxwell-scalar (EMS) models, described by the action

$$
\begin{aligned}
\mathcal{S}= & \frac{1}{4 \pi} \int d^{4} x \sqrt{-g} \\
& \left(\frac{R}{4}-\frac{f(\phi)}{4} F_{\mu \nu} F^{\mu \nu}-\frac{1}{2} \partial_{\mu} \phi \partial^{\mu} \phi\right),
\end{aligned}
$$

wherein the real scalar field $\phi$ has a canonical kinetic term and it is non-minimally coupled to the Maxwell field strength $F$, via some function $f(\phi)$, emerge naturally in physics ( $R$ is the Ricci scalar). Well known contexts are Kaluza-Klein models [2-6] and supergravity/string theory [7]. In these cases the

\footnotetext{
a e-mail: herdeiro@ua.pt

b e-mail: jmiguel.oliveira@ua.pt

c e-mail: eugen.radu@ua.pt
}

non-minimal coupling is provided by an exponential function of the sort $f(\phi) \sim e^{-\alpha \phi}$, where $\alpha$ is a constant. But more general classes of coupling functions have been considered, for instance, in the context of cosmology [8,9].

Another interesting class of coupling functions emerged recently in the quest for models that deviate from general relativity only in the strong gravity regime. If $f(\phi)$ obeys some simple properties, the EMS model accommodates the phenomenon of spontaneous scalarisation of asymptotically flat charged black holes [10]. This means that even though the electrovacuum Reissner-Nordström (RN) black hole is a solution of the EMS model, for sufficiently high charge to mass ratio this black hole becomes unstable: it becomes energetically favourable for the RN black hole to scalarise. A new family of scalarised black holes bifurcates from the RN family, which contains the end states of this dynamical scalarisation mechanism - see also [11-21].

The existence of such a class of EMS models, that allow spontaneous scalarisation of charged black holes, and accommodates two distinct families of black holes, the bald RN and the "hairy" scalarised BHs, raised the question if solitons can also be found in EMS models, as this is often the case in theories that accommodate both bald and hairy BHs. Building on this question, in [1] a set of theorems were established showing that, under various assumptions, no such solitonic solutions exist, similarly to the case of vacuum and electrovacuum. The purpose of this paper is to show that dropping one of the assumptions considered in [1], namely that the coupling function is everywhere finite, it becomes possible to circumvent the aforementioned theorems and obtain solitonic solutions, including in the flat spacetime (Maxwellscalar theory) limit. In the latter case, moreover, the solitons can be, in some examples, obtained in closed form. They yield pedagogical illustrations of how new physics could desingularise the Coulomb solution at the level of an effective field theory. In the self-gravitating case, the solutions are obtained numerically, although we cannot exclude that some 
carefully designed coupling functions exist where they will have a closed analytic form.

This paper is organised as follows. In Sect. 2 we discuss the flat spacetime analysis, i.e. the Maxwell-scalar model in Minkowski spacetime. We show that the model is integrable and how the Coulomb singularity can be regularised by a divergent coupling in a class of explicit examples. In Sect. 3 we consider the self-gravitating solitons, corresponding to the solutions discussed in flat spacetime in Sect. 2. Conclusions are presented in Sect. 4.

\section{Flat spacetime Maxwell-scalar models}

\subsection{A physical motivation}

An awkward feature of classical electromagnetism is that the energy $E$ of the Coulomb field of a point charge $Q$ is divergent:

$E \sim \int_{0}^{+\infty} \frac{Q^{2}}{r^{2}} d r=\infty$.

Quantum considerations naturally introduce an ultraviolet cut-off to the validity of the classical Coulomb solution, regularising this integral. Quantum Electrodynamics (QED), however, is itself incomplete as a quantum field theory, due to the Landau pole [22]. But it yields the important lesson that the coupling constant $g$, which determines the strength of the electromagnetic interaction in the Maxwell Lagrangian

$\mathcal{L}=-\frac{1}{4 g^{2}} F_{\mu \nu} F^{\mu \nu}$,

runs with the energy scale.

Whatever fundamental theory turns out to complete QED, it may admit a covariant effective field theory description that captures the dynamics of the coupling. Then, $g$ would emerge as a spacetime function with some dynamics. In a simple model, $g$ would be a real scalar field with a standard kinetic term. Allowing a more general dynamics, one takes $g$ as being an arbitrary function of the scalar field, keeping the latter with a standard kinetic term. This suggests considering the naive covariant effective field theory

$$
\begin{aligned}
\mathcal{S}= & \frac{1}{4 \pi} \int d^{4} x \sqrt{-g} \\
& \left(-\frac{f(\phi)}{4} F_{\mu \nu} F^{\mu \nu}-\frac{1}{2} \partial_{\mu} \phi \partial^{\mu} \phi\right),
\end{aligned}
$$

where $\phi$ is a real scalar field, $F=d A$ is the covariant description of the electromagnetic dynamics and the background is Minkowski spacetime. The function $f(\phi)$ specifies the dynamics of the gauge coupling. This model ignores higher order corrections in $F$, so it is certainly incomplete. Nonetheless one may take the aforementioned reasoning as a motiva- tion to consider this class of simple models. Can the Coulomb field of a point charge be de-singularised in this context?

\subsection{An integrable model}

The naive model (4), which is the decoupling limit of the EMS model (1) wherein back reaction is neglected, is integrable in the spherical sector. Taking the following ansatz for the fields in spherical coordinates in Minkowski spacetime $(t, r, \theta, \varphi)$ :

$\phi=\phi(r), \quad A=V(r) d t$

the Maxwell equations yield a first integral:

$V(r)=\int \frac{Q}{r^{2} f(\phi)} d r$,

where $Q$ is interpreted as the electric charge. Using this first integral, the Klein-Gordon equation reads

$r^{2} \frac{d}{d r}\left(r^{2} \frac{d \phi}{d r}\right)-\frac{Q^{2}}{2} \frac{d}{d \phi}\left(\frac{1}{f(\phi)}\right)=0$,

which, introducing the coordinate $x \equiv 1 / r$, yields another first integral

$$
\left(\frac{d \phi}{d x}\right)^{2}-\frac{Q^{2}}{f(\phi)}=\mathcal{E}
$$

It is a simple application of the virial theorem, or a Derricktype scaling theorem [23], to show that solutions must have $\mathcal{E}=0$. For instance, this can be seen from the condition [24]

$\int d^{3} x T_{i j}=0$

that holds for time-independent, finite energy field configuration in Minkowski spacetime, where $i, j$ are spatial indices in Cartesian coordinates. Relation (9) is a simple consequence of energy-momentum conservation and can be interpreted as the balancing of the total stresses in an extended object. There are regions where matter is in tension and regions where it is in compression, for any static balanced soliton. Thus, the problem of finding solutions is reduced to solving, from (8),

$x(\phi)=\frac{1}{Q} \int \sqrt{f(\phi)} d \phi$,

and then inverting $x(\phi) \rightarrow \phi(x) \rightarrow \phi(r)$. Fixing the coupling function $f(\phi)$ one can obtain $\phi(r)$ and, from (6), the electrostatic potential, both as line integrals. Due to the two first integrals the system is fully integrable. 


\subsection{Everywhere regular solutions}

To assess if the solutions have finite energy one must consider the energy-momentum of the model,

$$
\begin{aligned}
4 \pi T_{\mu \nu}= & f(\phi)\left(F_{\mu \alpha} F_{\nu}{ }^{\alpha}-\frac{1}{4} g_{\mu \nu} F_{\alpha \beta} F^{\alpha \beta}\right) \\
& +\partial_{\mu} \phi \partial_{\nu} \phi-\frac{1}{2} g_{\mu \nu} \partial_{\alpha} \phi \partial^{\alpha} \phi .
\end{aligned}
$$

This yields the energy density $\rho$, after using (8):

$\rho=T_{00}=\frac{Q^{2}}{4 \pi r^{4} f(\phi)}$.

and the total energy, $E$, obtained by integrating the energy density on a spacelike slice $\Sigma$

$E=\int_{\Sigma} \rho d^{3} x=\int_{0}^{+\infty} \frac{Q^{2}}{r^{2} f(\phi)} d r$.

In order to obtain regular solutions at the origin we assume the scalar field admits a power series expansion near the origin:

$\phi=\phi_{0}+\sum_{p=N} \phi_{p} r^{p}$,

We do not constrain the constant coefficient $\phi_{0}$, which may or may not vanish. Apart from $\phi_{0}$, let $\phi_{N}$, where $N \in \mathbb{N} \geqslant 1$ be the first non-vanishing coefficient in this expansion. Then, from (8),

$\left(-N r^{N+1} \phi_{N}+\ldots\right)^{2}=\frac{Q^{2}}{f(\phi)}$.

Thus, as $r \rightarrow 0$,

$f(\phi) \sim \frac{Q^{2}}{N^{2} \phi_{N}^{2}} \frac{1}{r^{2 N+2}}$.

Regularity of the scalar field at the origin then requires the coupling to diverge as $\sim 1 / r^{2 N+2}$. From (12) this implies that the energy density is finite therein and from (6),

$V(r)=V(0)+\frac{N^{2} \phi_{N}^{2}}{(2 N+1) Q} r^{2 N+1}+\ldots$,

close to the origin. Thus, all physical quantities are finite close to the origin, for this class of behaviours of the coupling.

\subsection{A class of examples}

There is still, of course, some freedom in choosing the coupling function, within the class with the correct divergent behaviour at the origin. Let us consider examples.

\subsubsection{A simple coupling yielding regular solutions}

As an explicit example, consider

$f(\phi)=\frac{1}{(1-\alpha \phi)^{4}}$,

where $\alpha$ is a non-zero constant. Then (10) immediately yields, taking the integration constant such that $\phi \rightarrow 0$ as $r \rightarrow \infty$ :

$\phi(r)=\frac{Q}{Q \alpha+r}$.

One observes that $\phi(r)$ is regular and smooth as $r \rightarrow 0$, $\phi(r) \simeq 1 / \alpha-r /\left(Q \alpha^{2}\right)$; thus we expect, from (16), that the coupling to diverge as $1 / r^{4}$. Asymptotically, on the other hand, $\phi(r) \simeq Q / r$. Thus the scalar "charge" coincides with the electric charge. Plugging (19) into (18) yields:

$f(r)=\left(1+\frac{\alpha Q}{r}\right)^{4}$.

The coupling diverges as $1 / r^{4}$ at the origin, as anticipated. This divergence precisely cancels the divergence of the Maxwell field at the origin, $c f$. (12), making it finite and non-zero. In fact, the energy density, from (12), is

$\rho=\frac{Q^{2}}{4 \pi(Q \alpha+r)^{4}}$.

It follows that the total energy (13) is

$E=\frac{Q}{3 \alpha}$.

Now, using (6) we obtain for the electrostatic potential:

$V(r)=-\frac{r Q}{(Q \alpha+r)^{2}}-\frac{\alpha^{2} Q^{3}}{3(Q \alpha+r)^{3}}$.

All the quantities (21), (22), (23) manifestly reduce to the usual Coulombic ones upon taking $\alpha \rightarrow 0$. In such case (19) reduces to the profile of a scalar charge $Q$ at the origin. The expressions make manifest how $\alpha$ regularises the solution.

\subsubsection{A family of couplings yielding regular solutions}

As further examples, with slightly different features, we generalise the coupling (18) as

$f(\phi)=\frac{1}{(1-\alpha \phi)^{n}}$,

where $n$ is an integer. Using this coupling, Eq. (10) gives

$\frac{1}{r}=\frac{1}{Q} \int(1-\alpha \phi)^{-n / 2} d \phi$,

which has a different indefinite integral for $n \neq 2$ and $n=2$. 
For $n \neq 2$, imposing $\phi(r \rightarrow \infty)=0$ to fix the integration constant, one obtains

$\phi(r)=\frac{1}{\alpha}-\frac{1}{\alpha}\left[1+\frac{\alpha Q(n-2)}{2 r}\right]^{\frac{2}{2-n}}$,

which reduces to (18) for $n=4$. For regular solutions at the origin we require $\lim _{r \rightarrow 0} \phi$ to be finite. This implies $n>2$, in which case

$\lim _{r \rightarrow 0} \phi(r)=\frac{1}{\alpha}-\frac{1}{\alpha}\left(\frac{2 r}{\alpha Q(n-2)}\right)^{\frac{2}{n-2}}$,

which is finite, as required. For $n=3$ we see that the second term goes as $r^{2}$; but for $n>4$, the second term has a noninteger power. In the former case we anticipate, from (16), that the coupling diverges as $1 / r^{6}$. In the latter case, $\phi$ is not analytic at the origin. It will, nonetheless yield a regular solution, when analysing the usual physical quantities.

The coupling $f(\phi)$ as a function of $r$ then reads:

$f(r)=\left[1+\frac{\alpha Q}{2 r}(n-2)\right]^{\frac{2 n}{n-2}}$,

which diverges as $\sim 1 / r^{\frac{2 n}{n-2}}$ at the origin, for $n>2$, but respects the condition $\lim _{r \rightarrow \infty} f(r)=1$. We confirm, in particular, the $1 / r^{6}$ divergence, for $n=3$ and a divergence with (generically) a non-integer inverse power for $n \geqslant 5$. The electric field $E_{\mu}=-\partial_{\mu} V(r)$ has only one non-zero component which reads, from (6)

$E_{r}(r)=-\frac{Q}{r^{2} f}=-\frac{Q}{r^{2}}\left[1+\frac{\alpha Q}{2 r}(n-2)\right]^{-\frac{2 n}{n-2}}$,

which behaves as $r^{\frac{4}{n-2}}$ near the origin, and it is thus regular for $n>2$.

The total energy now reads

$E=\frac{2 Q}{(n+2) \alpha}$

Thus, the family of cases with $n>2$ illustrate how regular solutions can be obtained, with a different analytic behaviour of the scalar field near the origin (the cases $n=3$ and $n=4$ ) and non-analytic behaviour $(n>4)$.

With $n=2$, following a similar reasoning one obtains

$\phi(r)=\frac{1}{\alpha}\left(1-e^{-\alpha Q / r}\right)$,

which is a regular solution at $r=0$ with $\lim _{r \rightarrow 0} \phi(r)=1 / \alpha$. The coupling function $f(\phi)$ becomes

$f(r)=e^{2 \alpha Q / r}$,

which, as before, also diverges at $r=0$ but respects $\lim _{r \rightarrow \infty} f(r)=1$. Observe, however, it does not diverge as an inverse power of $r$, which was the conclusion in Sect. 2.3.
This is because, again, $\phi$ in this case does not admit a power series expansion near the origin. This illustrates yet a different example of divergent coupling that yields regular solutions.

The electric field is now

$E_{r}(r)=-\frac{Q}{r^{2} f}=-\frac{Q}{r^{2}} e^{-2 \alpha Q / r}$,

and the total energy is

$E=\frac{Q}{2 \alpha}$

In these considerations $\alpha Q$ was assumed to be positive. Otherwise the total energy (30)-(34) would be negative, which would violate the weak energy condition. Interestingly enough, despite the seemingly different solution for $n=2$, the total energy $E$ is a smooth function of the power $n$, as (34) coincides with setting $n=2$ in (30).

2.5 Dilatonic coupling: a spherically symmetric solution

As mentioned in the Introduction, a dilatonic coupling

$f(\phi)=e^{-\alpha \phi}$,

where $\alpha$ is a constant, emerges in relevant scenarios. Let us thus briefly mention the existence of a spherically symmetric, exact solution for this coupling.

Considering (35) in (10), and taking the integration constant so that the scalar field vanishes asymptotically we immediately get

$\phi=-\frac{2}{\alpha} \log \left[1+\frac{\alpha Q}{2 r}\right]$.

Thus, the coupling, as a function of $r$ is

$f(\phi)=e^{-\alpha \phi}=\left[1+\frac{\alpha Q}{2 r}\right]^{2}$

Thus, the coupling diverges at the origin and, if $\alpha Q>0$ it is regular elsewhere. Moreover, using now (6) we get

$$
V(r)=-\frac{2 Q}{\alpha Q+2 r} .
$$

One finds the following small-r expansion of the solution

$$
\begin{aligned}
& \phi(r)=\frac{2}{\alpha}\left(\log r-\log \frac{\alpha Q}{2}\right)+\mathcal{O}(r), \\
& V(r)=-\frac{2}{\alpha}+\frac{4 r}{\alpha^{2} Q}+\mathcal{O}\left(r^{2}\right)
\end{aligned}
$$


thus, the scalar field diverges at the origin. Asymptotically, on the other hand, both fields are well behaved

$$
\begin{aligned}
& \phi(r)=-\frac{Q}{r}+\frac{\alpha}{4} \frac{Q^{2}}{r^{2}}+\mathcal{O}\left(\frac{1}{r^{3}}\right), \\
& V(r)=-\frac{Q}{r}+\frac{\alpha}{2} \frac{Q^{2}}{r^{2}}+\mathcal{O}\left(\frac{1}{r^{3}}\right) .
\end{aligned}
$$

The energy density of this solution diverges at the origin:

$\rho=-T_{t}^{t}=\frac{Q^{2}}{\pi r^{2}(\alpha Q+2 r)^{2}} ;$

the total mass, however, is finite

$M=4 \pi \int_{0}^{\infty} d r r^{2} \rho=\frac{2 Q}{\alpha}$.

This solution is interesting in that it shows a divergent coupling can source a finite mass configuration which, nonetheless, is not fully regular, as the scalar field and the energy density diverge at the origin.

\section{The gravitating solitons}

The above flat spacetime solutions can be made to selfgravitate by coupling (4) to Einstein's general relativity. For the case of the regular solutions described in the previous section, this yields, perhaps, the simplest models of charged soliton.

One now considers the EMS model (1), where $c=G=1$. In addition to the ansatz (5) we consider the metric form

$$
\begin{aligned}
& d s^{2}=-e^{-2 \delta(r)} N(r) d t^{2}+\frac{d r^{2}}{N(r)}+r^{2}\left(d \theta^{2}+\sin ^{2} \theta d \varphi^{2}\right), \\
& \text { where } N(r) \equiv 1-\frac{2 m(r)}{r},
\end{aligned}
$$

and $m(r)$ is the Misner-Sharp mass; $r$ is thus the areal radius, a geometrically meaningful coordinate.

The ansatz (5) and (43) yield the following effective Lagrangian:

$$
\mathcal{L}_{\text {eff }}=e^{-\delta} m^{\prime}-\frac{r^{2}}{2} e^{-\delta} N \phi^{\prime 2}+\frac{r^{2}}{2} f(\phi) e^{\delta} V^{\prime 2} .
$$

As in the flat spacetime case, the equation of the electric potential possesses a first integral, which generalises (6), and reads

$V^{\prime}=e^{-\delta} \frac{Q}{r^{2} f(\phi)}$,

where again the integration constant $Q$ is the electric charge, which we shall assume to be strictly positive, without any loss of generality. Using this integral, the remaining equations of motion become ${ }^{1}$

$$
\begin{aligned}
& m^{\prime}=\frac{r^{2}}{2} N \phi^{\prime 2}+\frac{Q^{2}}{2 r^{2}} S(\phi), \\
& \delta^{\prime}+r \phi^{\prime 2}=0, \\
& \left(e^{-\delta} r^{2} N \phi^{\prime}\right)^{\prime}-\frac{e^{-\delta}}{2 r^{2}} \frac{d S(\phi)}{d \phi} Q^{2}=0,
\end{aligned}
$$

where we have defined

$S(\phi) \equiv \frac{1}{f(\phi)}$.

The smooth of a spacetime configurations can be assessed by analysing the Ricci scalar

$$
\begin{aligned}
R= & \frac{N}{r}\left(3 r \delta^{\prime}-4\right) \\
& +\frac{2}{r^{2}}\left[1+N\left(r^{2} \delta^{\prime \prime}-\left(1-r \delta^{\prime}\right)^{2}\right)\right]-N^{\prime \prime},
\end{aligned}
$$

and the Kretschmann scalar

$$
\begin{aligned}
K= & \frac{4}{r^{4}}(1-N)^{2}+\frac{2}{r^{2}}\left[N^{\prime 2}+\left(N^{\prime}-2 N \delta^{\prime}\right)^{2}\right] \\
& +\left[N^{\prime \prime}-3 \delta^{\prime} N^{\prime}+2 N\left(\delta^{\prime 2}-\delta^{\prime \prime}\right)\right]^{2}
\end{aligned}
$$

\subsection{Asymptotic expansions}

\subsubsection{Near the origin}

A small $r$ analysis of the field equations confirms the conclusion observed in the flat space analysis: for a scalar field admitting a power series expansion near the origin $\phi=\phi_{0}+\phi_{1} r+\ldots$ and $\phi_{1} \neq 0$, if the coupling diverges as $1 / r^{4}$, finite energy, everywhere regular solutions are possible. To see this, we again start by assuming the existence of a power series expansion of solutions, with the scalar field approaching a finite nonzero value

$\phi(r) \rightarrow \phi_{0}$ as $r \rightarrow 0$

where $\phi_{0}$ is arbitrary. Then, the equations of motion, together with the assumption of regularity, impose, for the $n^{\text {th }}$ derivative of $S(\phi)$ computed at the origin, denoted $S^{(n)}\left(\phi_{0}\right)$,

$$
\begin{aligned}
& S\left(\phi_{0}\right)=S^{(1)}\left(\phi_{0}\right)=S^{(2)}\left(\phi_{0}\right)=S^{(3)}\left(\phi_{0}\right)=0, \\
& \quad \text { whereas } \quad S^{(4)}\left(\phi_{0}\right)>0 .
\end{aligned}
$$

This implies the advertised behaviour: the coupling function $f(\phi)$ diverges as $1 / r^{4}$ as $r \rightarrow 0$. This behaviour cancels the divergence associated with the presence of an electric charge, providing a smooth configuration as $r \rightarrow 0$.

\footnotetext{
1 There is an extra equation, which is a constraint and can be derived from (46)-(48).
} 
The small $r$ expansion of the matter functions reads

$\phi(r)=\phi_{0}-\frac{2 \sqrt{6} r}{Q \sqrt{S^{(4)}\left(\phi_{0}\right)}}+\phi_{2} r^{2}+\ldots$,

$V(r)=-\frac{8 e^{-\delta_{0}}}{Q^{3} S^{(4)}\left(\phi_{0}\right)} r^{3}+\ldots$,

while for the metric functions we find

$$
\begin{aligned}
m(r) & =\frac{8}{Q^{2}} \frac{1}{S^{(4)}\left(\phi_{0}\right)} r^{3}-\frac{2 \sqrt{6} \phi_{2}}{Q \sqrt{S^{(4)}\left(\phi_{0}\right)}} r^{4}+\ldots, \\
\delta(r) & =\delta_{0}-\frac{12 r^{2}}{Q^{2} S^{(4)}\left(\phi_{0}\right)}+\ldots,
\end{aligned}
$$

where $\phi_{2}$ and $\delta_{0}$ are constants that are fixed by the numerics when integrating the field equations from the origin to infinity and requiring the correct asymptotic behaviour. With this expansion, both the Kretschmann curvature scalar and Ricci scalar are finite as $r \rightarrow 0$, taking the form

$$
\begin{aligned}
K & \equiv R_{\mu \nu \alpha \beta} R^{\mu \nu \alpha \beta} \\
& =\frac{3840}{Q^{4}\left[S^{(4)}\left(\phi_{0}\right)\right]^{2}}-\frac{2560 \sqrt{6} \phi_{2}}{Q^{3}\left[S^{(4)}\left(\phi_{0}\right)\right]^{3 / 2}} r+\ldots,
\end{aligned}
$$

and

$R=\frac{48}{Q^{2} S^{(4)}\left(\phi_{0}\right)}-\frac{16 \sqrt{6} \phi_{2}}{Q\left(S^{(4)}\left(\phi_{0}\right)\right)^{1 / 2}} r+\ldots$

The small $r$ expansion of $S(\phi)$ reads

$S(\phi)=\frac{24}{Q^{4} S^{(4)}\left(\phi_{0}\right)} r^{4}-\frac{8 \sqrt{6} \phi_{2}}{Q^{3} \sqrt{S^{(4)}\left(\phi_{0}\right)}} r^{5}+\ldots$,

which implies the following generic approximate form of the coupling function

$S(\phi)=\frac{1}{f(\phi)} \sim\left(\phi-\phi_{0}\right)^{4}$ as $r \rightarrow 0$.

Of course, we could have assumed that in the scalar field expansion $\phi_{1}=0$ and the power series starts at a higher order term. This would impact in the way the coupling diverges at the origin, similarly to the flat spacetime analysis of Sect. 2.3. For concreteness, here we focus on the case with $\phi_{1} \neq 0$. This case corresponds, in the non-back-reacting case, to having $N=1$ in Eq. (14). Choosing $\phi_{1}=0$ in the latter would imply a different behaviour for the divergence of $f(\phi)$, implied by the Eq. (16). In the back-reacting case this would correspond to having $S^{(4)}\left(\phi_{0}\right)=0$. Non-trivial solutions with such behaviour should exist, as well.

\subsubsection{Near infinity}

A large $r$ analysis of the field equations, on the other hand, imposing

$f(\phi) \rightarrow 1$ as $r \rightarrow \infty$ yields the following approximate solutions:

$m(r)=M-\frac{Q^{2}+Q_{s}^{2}}{2 r}+\ldots$,
$\phi(r)=\frac{Q_{s}}{r}+\ldots$,
$V(r)=\Phi-\frac{Q}{r}+\ldots, \quad \delta(r)=\frac{Q_{s}^{2}}{2 r^{2}}+\ldots$

Here $M$ is the ADM mass and $Q$ is the electric charge; $\Phi$ is the electrostatic potential at infinity and $Q_{s}$ is the scalar 'charge' which in general needs not equal the electric charge (it did in the flat spacetime illustration above). In fact, the equations of motion possess again two first integrals implying that the gravitating solutions satisfy the following relation

$M^{2}+Q_{s}^{2}=Q^{2}$.

This last equation, in particular, shows manifestly the curved background breaks the equality between $Q$ and $Q_{s}$.

Interestingly, one can show that there is a Smarr relation in terms of these asymptotic quantities, which is not affected by the scalar field,

$M=\Phi Q$.

Moreover, a first law of thermodynamics can be obtained in the form

$d M=\Phi d Q$

We emphasise the absence of a scalar field contribution in these relations.

\subsection{The full solutions}

The gravitating version of the exact solution in Minkowski spacetime described in Sect. 2.4.1, with coupling (18), and whose asymptotic limits have been described in Sect. 3.1, can be constructed numerically. The set of four ordinary differential equations obtained from the above setup was solved numerically by using a standard Runge-Kutta ordinary differential equation solver and appropriate boundary conditions. Fixing $\alpha$, gravitating solitons exist for arbitrary large values of $Q$. The profile of a typical solution is shown in Fig. 1. As one can see, the profiles of the various functions, and in particular that of the Kretschmann scalar $K$, are smooth as $r \rightarrow 0$. In Fig. 2 we show the ADM mass $v s$. electric charge diagram for families of solutions with different values of $\alpha$. One can see that for all families, the solutions trivialise as $\alpha \rightarrow 0$. Moreover, a smaller $\alpha$ implies that the same value of the electric charge can support a more massive soliton. Obviously, the solutions also trivialise as $Q \rightarrow 0$. The electric charge supports the soliton. This is also manifest from the following virial identity that can be derived for these solutions: 


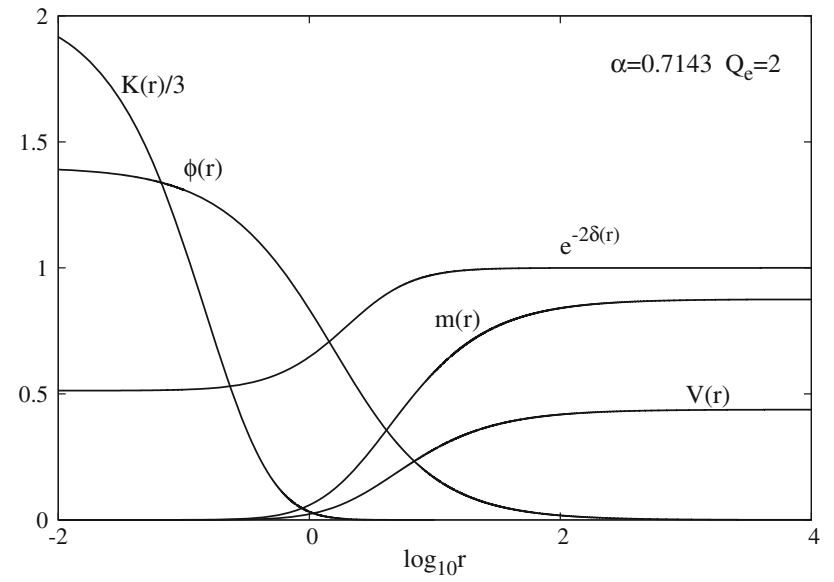

Fig. 1 Profiles of an illustrative gravitating soliton with the coupling (18)

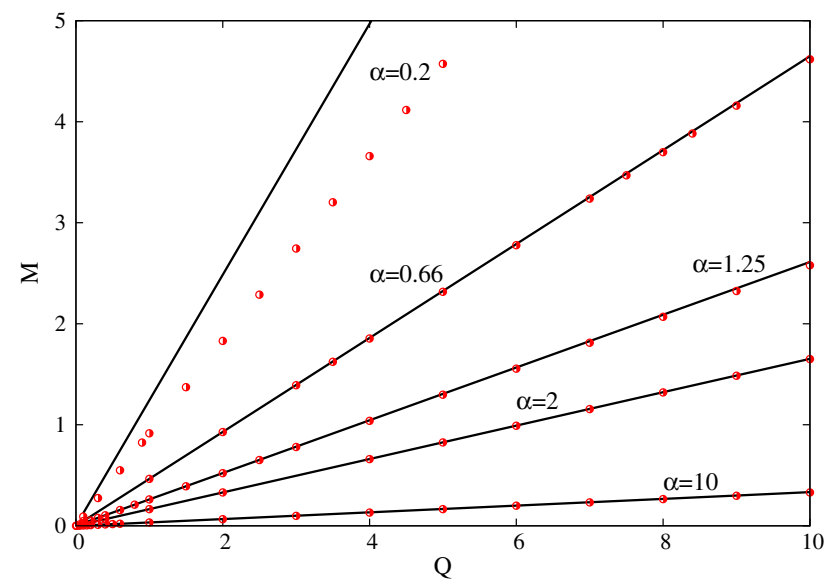

Fig. 2 ADM $v s$. electric charge for families of gravitating solitons with different values of $\alpha$. The straight lines are obtained from the perturbative solutions, whereas the dots represent the numerical solutions

$\int_{0}^{\infty} d r e^{-\delta} \phi^{\prime 2}=\int_{0}^{\infty} d r \frac{e^{-\delta}}{r^{2}} \frac{Q^{2}}{f(\phi)}$.

For $Q=0$ the right hand side vanishes, and so must the left hand side, which implies $\phi^{\prime}=0$ and hence no non-trivial scalar profile exists.

Self-gravitating solitons with the coupling (24) and $n=3$ where also obtained. They follow the same pattern as the $n=3$ case, which is therefore illustrative.

\subsection{Perturbative solutions}

The existence of a flat spacetime solution, whose total mass-energy is proportional to $1 / \alpha$, suggests that the selfgravitating solitons may be expressed as a perturbative series in $1 / \alpha$. Let us indeed show that the numerical solutions of the previous subsection can be approximated by such perturbative solutions. This approximation, as we will show and as one may anticipate, is accurate for sufficiently large $\alpha$.
The perturbative solutions are obtained by performing a power series expansion for all relevant functions

$m(r)=\sum_{k \geqslant 1}\left(\frac{1}{\alpha}\right)^{k} m_{k}(r)$,
$\delta(r)=\sum_{k \geqslant 1}\left(\frac{1}{\alpha}\right)^{k} \delta_{k}(r)$,
$\phi(r)=\sum_{k \geqslant 1}\left(\frac{1}{\alpha}\right)^{k} \phi_{k}(r)$,
$V(r)=\sum_{k \geqslant 1}\left(\frac{1}{\alpha}\right)^{k} V_{k}(r)$.

As for the numerical solutions of the previous subsection, we focus on the quartic coupling function (18). Solving iteratively the field equations order by order in $1 / \alpha$, we arrive at the following expressions ${ }^{2}$

$$
\begin{aligned}
& m_{1}(r)=0=m_{3}(r), \quad m_{2}(r)=\frac{q r^{3}}{3(q+r)^{3}} \\
& m_{4}(r)=-\frac{q r^{4}\left(10 q^{2}+q r+r^{2}\right)}{90(q+r)^{6}} \\
& \delta_{1}(r)=0=\delta_{3}(r), \quad \delta_{2}(r)=\frac{q^{2}(q+3 r)}{6(q+r)^{3}} \\
& \delta_{4}(r)=\frac{q^{2}\left(q^{4}+6 q^{3} r+15 q^{2} r^{2}+100 q r^{3}-30 r^{4}\right)}{540(q+r)^{6}} \\
& \phi_{1}(r)=\frac{q}{q+r}, \quad \phi_{2}=0, \quad \phi_{3}(r)=\frac{q(2 q-r) r^{2}}{18(q+r)^{4}} \\
& V_{1}(r)=\frac{r^{3}}{3(q+r)^{3}}, \quad V_{2}(r)=0 \\
& V_{3}(r)=-\frac{r^{3}\left(5 q^{3}+25 q^{2} r+6 q r^{2}+r^{3}\right)}{90(q+r)^{6}}
\end{aligned}
$$

where $q$ is a free parameter, whose physical significance becomes transparent by computing the far field asymptotics of the electric potential. One finds it is related to the electric charge measured at infinity $Q$, as

$$
Q=\frac{q}{\alpha} \text {. }
$$

The perturbative solution yields the following ADM mass and scalar charge, valid to fourth order in perturbation theory:

$$
\begin{aligned}
& M=\frac{Q}{3 \alpha}\left(1-\frac{1}{30 \alpha^{2}}+\frac{1}{1080 \alpha^{4}}\right) \\
& Q_{s}=\left(1-\frac{1}{18 \alpha^{2}}+\frac{7}{3240 \alpha^{4}}\right) Q
\end{aligned}
$$

\footnotetext{
$\overline{2}$ We have computed the solution up to eighth order and no obvious pattern could be found. Here we display only the first few terms for each function.
} 
Observe that the first terms in (71) reproduce the flat spacetime limit, Eq. (22) and the fact that the electric and scalar charge coincide in that limit.

In Fig. 2 the perturbative solutions (71) are compared with the numerical solutions. One can observe that the former provide a good approximation for large values of $\alpha$; for instance, for $\alpha=10$ the relative difference between the numerical result for $M(Q)$ and the theory one is around $10^{-4}$. However, the differences start to increase for smaller $\alpha$. This is illustrated by the results for $\alpha=0.2$ in Fig. 2 .

Finally let us mention that a similar solution has been derived for the self-gravitating solitons with the coupling (24) and $n=3$. In this case one finds, e.g.

$M=\frac{2 Q}{5 \alpha}\left(1-\frac{2}{45 \alpha^{2}}+\frac{22}{14625 \alpha^{4}}\right)+\ldots$

\section{Discussion}

Recently, a set of theorems were shown [1] establishing that the model (1) does not allow self-gravitating solitons. One of the observations therein is that if the coupling would diverge the theorems could, potentially, be circumvented. The purpose of this paper was to provide the mechanism how this can happen providing a simple construction of flat spacetime and gravitating solitons.

Preliminary analysis shows the solitons we have described herein are stable against spherical perturbations. If this is the case for generic perturbations, these solitons can be used for dynamical studies in many setups, as, for instance, boson stars $[25,26]$. Moreover, this construction reveals how to desingularise the Coulomb field in a classical field theory, without resorting to non-linear electrodynamics, as in the BornInfeld model [27], or invoking a manifestly extended object, such as in the Dirac model of the electron as a spherical membrane [28].

Finally, let us remark that there is a well known similarity between the EMS model and the extended scalartensor-Gauss-Bonnet model, where black hole scalarisation was first pointed out in [29-31]. Very recently, a family of particle-like solutions in the latter model were discussed [32]. These particle like solutions are also supported by a divergent coupling making them the counterparts of the solutions described herein. But in the cases reported in [32] the scalar field also diverges at the origin, in contrast with our fully regular solutions.

Acknowledgements J.O. is supported by the FCT Grant PD/BD/1281 $84 / 2016$. This work is supported by The Center for Research and Development in Mathematics and Applications (CIDMA) and by the Centre of Mathematics (CMAT) through the Portuguese Foundation for Science and Technology (FCT - Fundação para a Ciência e a Tecnologia), references UIDB/04106/2020, UIDP/04106/2020, UIDB/00013/2020 and UIDP/00013/2020 and by national funds (OE), through FCT, I.P., in the scope of the framework contract foreseen in the numbers 4, 5 and 6 of the article 23, of the Decree-Law 57/2016, of August 29, changed by Law 57/2017, of July 19. We acknowledge support from the project PTDC/FIS-OUT/28407/2017. This work has further been supported by the European Union's Horizon 2020 research and innovation (RISE) programmes H2020-MSCA-RISE2015 Grant no. StronGrHEP-690904 and H2020-MSCA-RISE-2017 Grant no. FunFiCO-777740. The authors would like to acknowledge networking support by the COST Action CA16104.

Data Availability Statement This manuscript has no associated data or the data will not be deposited. [Authors' comment: There is no data to be uploaded since this is a theoretical study.]

Open Access This article is licensed under a Creative Commons Attribution 4.0 International License, which permits use, sharing, adaptation, distribution and reproduction in any medium or format, as long as you give appropriate credit to the original author(s) and the source, provide a link to the Creative Commons licence, and indicate if changes were made. The images or other third party material in this article are included in the article's Creative Commons licence, unless indicated otherwise in a credit line to the material. If material is not included in the article's Creative Commons licence and your intended use is not permitted by statutory regulation or exceeds the permitted use, you will need to obtain permission directly from the copyright holder. To view a copy of this licence, visit http://creativecomm ons.org/licenses/by/4.0/.

Funded by $\mathrm{SCOAP}^{3}$.

\section{References}

1. C.A.R. Herdeiro, J.M.S. Oliveira, Class. Quantum Gravity 36(10), 105015 (2019). arXiv:1902.07721 [gr-qc]

2. T. Kaluza, Sitzungsber. Preuss. Akad. Wiss. Berlin (Math. Phys. ) 1921, 966 (1921)

3. T. Kaluza, Int. J. Mod. Phys. D 27(14), 1870001 (2018). arXiv:1803.08616 [physics.hist-ph]

4. O. Klein, Z. Phys. 37, 895 (1926)

5. O. Klein, Surv. High Energy Phys. 5, 241 (1986)

6. T. Appelquist, A. Chodos, P.G.O. Freund, Reading, USA: AddisonWesley (1987) 619 (Frontiers in physics, 65)

7. P. Van Nieuwenhuizen, Phys. Rep. 68, 189 (1981)

8. J. Martin, J. Yokoyama, JCAP 0801, 025 (2008). arXiv:0711.4307 [astro-ph]

9. A. Maleknejad, M.M. Sheikh-Jabbari, J. Soda, Phys. Rep. 528, 161 (2013). arXiv:1212.2921 [hep-th]

10. C.A.R. Herdeiro, E. Radu, N. Sanchis-Gual, J.A. Font, Phys. Rev. Lett. 121(10), 101102 (2018). arXiv:1806.05190 [gr-qc]

11. P.G.S. Fernandes, C.A.R. Herdeiro, A.M. Pombo, E. Radu, N. Sanchis-Gual, Class. Quantum Gravity 36(13), 134002 (2019). arXiv:1902.05079 [gr-qc]

12. D. Astefanesei, C. Herdeiro, A. Pombo, E. Radu, JHEP 10, 78 (2019). arXiv:1905.08304 [hep-th]

13. Y.S. Myung, D.C. Zou, Eur. Phys. J. C 79(3), 273 (2019). arXiv: 1808.02609 [gr-qc]

14. Y.S. Myung, D.C. Zou, Phys. Lett. B 790, 400 (2019). arXiv: 1812.03604 [gr-qc]

15. Y.S. Myung, D.C. Zou, Eur. Phys. J. C 79(8), 641 (2019). arXiv:1904.09864 [gr-qc]

16. R.A. Konoplya, A. Zhidenko, Phys. Rev. D 100(4), 044015 (2019). arXiv: 1907.05551 [gr-qc]

17. P.G.S. Fernandes, C.A.R. Herdeiro, A.M. Pombo, E. Radu, N. Sanchis-Gual, Phys. Rev. D 100, 084045 (2019). arXiv: 1908.00037 [gr-qc] 
18. M. Boskovic, R. Brito, V. Cardoso, T. Ikeda, H. Witek, Phys. Rev. D 99, 035006 (2019). arXiv: 1811.04945 [gr-qc]

19. M. Minamitsuji, T. Ikeda, Phys. Rev. D 99, 04401 (2019). arXiv:1812.03551 [gr-qc]

20. H.O. Silva, C.F.B. Macedo, T.P. Sotiriou, L. Gualtieri, J. Sakstein, E. Berti, Phys. Rev. D 99, 064011 (2019). arXiv:1812.05590 [grqc]

21. C.A.R. Herdeiro, E. Radu, Phys. Rev. D 99(8), 084039 (2019). arXiv:1901.02953 [gr-qc]

22. M.E. Peskin, D.V. Schroeder, An Introduction to Quantum Field Theory (Taylor \& Francis Inc., New York, 1995)

23. G.H. Derrick, J. Math. Phys. 5, 1252 (1964)

24. G.W. Gibbons, Lect. Notes Phys. 383, 110 (1991). arXiv: 1109.3538 [gr-qc]

25. S.L. Liebling, C. Palenzuela, Living Rev. Relativ. 15, 6 (2012)

26. S.L. Liebling, C. Palenzuela, Living Rev. Relativ. 20(1), 5 (2017). arXiv:1202.5809 [gr-qc]
27. M. Born, L. Infeld, Proc. R. Soc. Lond. A 144(852), 425 (1934)

28. P.A.M. Dirac, Proc. R. Soc. Lond. A 268, 57 (1962)

29. D.D. Doneva, S.S. Yazadjiev, Phys. Rev. Lett. 120(13), 131103 (2018). arXiv:1711.01187 [gr-qc]

30. H.O. Silva, J. Sakstein, L. Gualtieri, T.P. Sotiriou, E. Berti, Phys. Rev. Lett. 120(13), 131104 (2018). arXiv:1711.02080 [gr-qc]

31. G. Antoniou, A. Bakopoulos, P. Kanti, Phys. Rev. Lett. 120(13), 131102 (2018). arXiv:1711.03390 [hep-th]

32. B. Kleihaus, J. Kunz, P. Kanti, arXiv:1910.02121 [gr-qc] 